using an apparatus with less efficient mixing; it was for this reason that the results were considered of insufficient precision for the calculation of $B_{12}$. However, since in all cases $x_{a}{ }^{\prime}<x_{2}$, a negative value of $B_{12}$ was indicated, in keeping with the predictions of the theory of corresponding states.

To calculate a value of $B_{12}$ from all data equation (7) was used in conjunction with Coggan's ${ }^{5}$ multi-fit programme using the Atlas I computer at the University of Manchester. In terms of the criterion

$$
\Sigma\left(x_{2}{ }^{\text {observed }}-x_{2} \text { calculated }\right)^{2}
$$

the best value for each set of data, namely -997 for dinonyl phthalate and -540 for squalane, was approximately three times better than the corresponding states value of -158 . The value -790 which is five times the theoretical value of $B_{12}$ appoars to give a reasonable fit to both systems, particularly when $P-p_{2}$ approaches $1 \mathrm{~atm}$., and is therefore suggested for calculations in gas-liquid chromatography. There is evidence, however, that structural alterations in the mathematical model would enable a very much moro accurate fit of data to be made. The theoretical implications of such a model are at present being examined by Dr. Coggan and myself.

I thank Dr. G. C. Coggan for his help in connexion with the data processing and Mr. P. A. Sewell and Dr. R. Stock for making all their results available.

Department of Chemical Engineering, University of Nottingham.

Sewell, P. A., and Stock, R., Nature, 20\%, 618 (1965).

2 Freeguard, G. F., thesis, Univ. Wales (1961).

3 Freeguard, G. F., and Stock, R., Trans. Faraday Soc., 59, 1655 (1963)

${ }^{4}$ Sewell, P. A., and Stock, R. (personal communication).

Coggan, G. C. (to be published).

\section{Joule-Thomson Effect : Non-ideality and Association}

IT is generally asserted in text-books on thermodynamics and the properties of gases that the JouleThomson effect demonstrates conclusively that real gases are non-ideal. This is indeed the case, but not in the sense that usually seems to be understood.

The Joule-Thomson effect is a measure of the extent to which, at constant pressure, the volume of a fixed mass of gas fails to be strictly proportional to the absolute temperature. Such departures from the relation $p V \propto T$ occur in: (i) non-ideal gases in which there is no chemical reaction; (ii) ideal quantum gases at very low temperatures; (iii) associating gases (ideal and non-ideal).

Case (ii) has been considered briefly by Landsberg.' The purpose of the present communication is to emphasize the relevance of case (iii) (exemplified by the large ${ }^{2}$ JouleThomson effect exhibited in experiments ${ }^{3}$ conducted for another purpose on the associating gas $2 \mathrm{NO}_{2} \rightleftharpoons \mathrm{N}_{2} \mathrm{O}_{4}$ ); and to point out that, even when the possibility of association is taken into account, it is still possible to use exporimental observations on the Joule-Thomson effect as one of the sources of compelling evidence of nonideality.

Now whereas for inert non-ideal gases there may be either a decrease or an increase in temperature, in an ideal associating gas the Joule-Thomson expansion always leads to a decrease in temperature. That is, $(\partial T / \partial p)_{H}=-(\partial H / \partial p)_{T} / C_{p}$ is always positive (but, provided that a dimer is one of the associated species present, resembles the value for an inert non-ideal gas in tending to a non-zero limit at low pressures). For increase in pressure at constant temperature leads to increased association, and therefore, since enthalpies of dissociation are positive, to decrease in $H$. Thus, $(\partial H / \partial p)_{T}$ for an ideal associating gas is always negative, which implies that $(\partial T / \partial p)_{H}$ is always positive.

It is found from experiment that in certain cases $(\partial T / \partial p)_{H}$ is negative, as for hydrogen and helium at room temperature and atmospheric pressure, which compels the conclusion that these systems are non-ideal, and cannot be ideal associating gases. That is: the Joule-Thomson effect does not of itself demonstrate non-ideality (as distinct from association); but inversion of the JouleThomson effect does.

This conclusion is akin to one concerning empirical equations of state. If at low pressures an expression $p V=R T+B p$ is found to apply, a negative value of $B$ might be attributable to association, to non-ideality, or to both together; but a positive value of $B$ cannot occur for an ideal associating gas, and so is conclusive proof of non-ideality.

The point considered here is one more example of similarities in the effects of non-ideality and of chemical reaction (compare, for example, "deviations from Nernst's distribution law"). It should be emphasized that in particular instances, as indicated here, it is possible to show conclusively that the system considered is non-ideal.

I thank Prof. P. Gray for valuable discussion of these matters. This communication was prepared during the tenure of the Edward A. Deeds fellowship of Queen's College, Dundee.

Chemistry Department,

P. G. Wright

Queen's College, Dundee.

${ }^{2}$ Landsberg, P. T., Thermodynamics with Quantum Statistical Illustrations, 73, 203 (New York, 1961).

${ }^{2}$ Partington, J. R., An Advanced Treatise on Physical Chemistry, 1, 615 (London, 1949).

${ }^{3}$ Brass, P. D., and Tolman, R. C., J. Amer. Chem. Soc., 54, 1003 (1932).

\section{Effect of Interstitial Atoms on a Lattice- vacancy Diffusional Process}

AN investigation has been made of the mechanism of diffusion of chromium into iron-carbon systems over the temperature range $950^{\circ}-1,265^{\circ} \mathrm{C}$. At temperatures above $825^{\circ} \mathrm{C}$, the diffusional mechanism of chromium into iron is complicated by a change in crystal structure from a body-centred cubic lattice ( $\alpha$-phase) to a face-centred lattice ( $\gamma$-phase). At low concentrations of carbon, a lattice-vacancy diffusional mechanism has been postulated, and a moving boundary model set up to represent this condition $^{1}$. The results have confirmed that at concentrations of carbon of less than $0 \cdot 16$ per cent only latticevacancy diffusion was effective. At concentrations of carbon above this, the interstitial atoms hinder diffusion.

This communication presents results illustrating this effect for concentrations of carbon up to 1 per cent (Table 1).

$$
\text { Fig. 1, a plot of } \frac{\text { diffusivity of chromium in iron ( } \gamma \text {-phase })}{\text { diffusivity of chromium in iron ( } \alpha \text {-phase) }}
$$

versus concentration of carbon, shows that for concentrations of carbon up to 0.16 per cent this ratio is independent

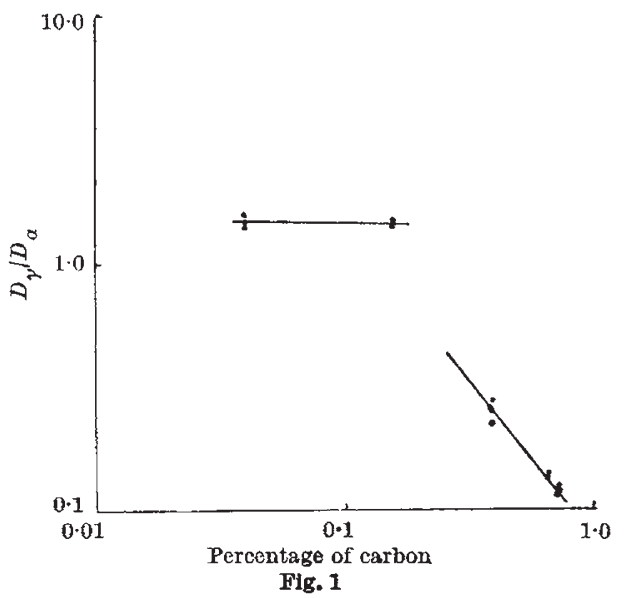

